\title{
Understanding Micropolar Theory in the Earth Sciences II: The Seismic Moment Tensor
}

\author{
Rafael Abreu ${ }^{1}$ (D) and Stephanie Durand ${ }^{2}$
}

\begin{abstract}
Seismic events produced by block rotations about vertical axis occur in many geodynamic contexts. In this study, we show that these rotations can be accounted for using the proper theory, namely micropolar theory, and a new asymmetric moment tensor can be derived. We then apply this new theory to the Kaikōura earthquake (2016/11/14), Mw 7.8, one of the most complex earthquakes ever recorded with modern instrumental techniques. Using advanced numerical techniques, we compute synthetic seismograms including a full asymmetric moment tensor and we show that it induces measurable differences in the waveforms proving that seismic data can record the effects of the block rotations observed in the field. Therefore, the theory developed in this work provides a full framework for future dynamic source inversions of asymmetric moment tensors.
\end{abstract}

Keywords: Seismology, asymmetric moment tensor, micropolar theory, Kaikōura earthquake.

\section{Introduction}

The main deformation observed on continents is generated by the contemporaneous motion of numerous strike-slip faults (Schreurs, 1994). Strikeslip faults are vertical or nearly vertical fractures where the tectonic blocks have mostly moved horizontally. Therefore, in many tectonically deformed areas of the crust, distributed deformation is manifested as a broad region of faulting (McKenzie \& Jackson, 1986). The motion of a system of strike slip faults is a very efficient mechanism of large-scale deformation (Twiss et al., 1993).

In continental regions, the rupture lengths of even the largest earthquakes are often small compared with

1 Institut für Geophysik, Westfälische Wilhelms-Universität Münster, Corrensstraße 24, 48149 Münster, Germany. E-mail: abreu@uni-muenster.de

2 Univ Lyon, UCBL, ENSL, UJM, CNRS, LGL-TPE, 69622 Villeurbanne, France. the width of the deforming zone (Jackson and McKenzie, 1988). Such zones of distributed continental deformation frequently contain fault-bounded blocks that rotate rapidly with respect to the major plates on either side (Kissel et al., 1985, 1986; Luyendyk et al., 1980, 1985; Walcott 1984). The study of general features of the deformation induced by systems of sub-parallel strike-slip faults shows that the governing constraints are kinematic: the fault blocks must remain in contact with each other and the deformed area must fit with its surroundings. As a result, fault blocks that move laterally without significant internal deformation also rotate about vertical axis relative to boundaries of the fault domain by an amount that is quantitatively related to the fault slip, spacing and orientation (Freund, 1970; Garfunkel, 1974; Garfunkel \& Ron, 1985).

Rotation of the blocks and their relative lateral motion are two different but essential and contemporaneous expressions of a single deformation. Like motions of large tectonic plates, the motion of smaller blocks at the surface of the Earth can be described by rotations about vertical axis (Euler poles) that intersect the center of the Earth. To the eye, these rotations produce gradients in the surface velocity field that could be mistaken for shear rates because both involve the components of the velocity gradient tensor (McCaffrey et al., 2002). Many observed GPS velocity fields can be explained by rotating blocks that are strained near their edges due to interactions with other blocks across the bounding faults. The rotation of fault-bounded domains results in sigmoidal antithetic faults that have a dip-slip component and a dip direction that changes along the strike (Schreurs, 1994).

Block rotation is also an important process in fault gouges (fault in rocks formed by tectonic forces with a very small grain size) (Alonso-Marroquin et al., 
2006). Traditionally, fault movement is treated with standard continuum mechanic formulations, where faults are modeled as discontinuities within a velocity field. This over simplification makes it difficult to determine e.g., fault-weakening processes (AlonsoMarroquin et al., 2006; Collins-Craft et al., 2020; Rattez et al., 2018a, b; Veveakis et al., 2012). Veveakis et al. (2012) used micropolar theory to model fault gouge and they were able to show that thermal effects foster the localization of deformation with the formation of very thin shear bands. In more recent works (Collins-Craft et al., 2020; Rattez et al., 2018a, b), micropolar theory was used to investigate the evolution of the localization zone thickness with respect to the evolution of the grain size. Addessi (2014) pointed out that the micropolar approach is also suitable to model brittle-like materials that have a strain softening constitutive behavior which can be observed in mature fault zones (Addessi, 2014; Brandes \& Tanner, 2020, Sibson, 1984). The fact that the micropolar theory allows rotational motions makes it especially useful to describe fault creep, where particle rotation is a possible explanation for the gradual fault movement (Brandes \& Tanner, 2020).

Natural examples of fault and block rotations about vertical axis occur in many areas of the planet such as the San Andreas fault system in southern California (Nicholson et al., 1986a, b; Schreurs, 1994), eastern Iran (Freund, 1970), New Zealand (Roberts, 1995; Ron et al., 1984; Walcott, 1984; Wang et al., 2020; ), the Dead Sea transform (Le Pichon \& Gaulie, 1988; Ron \& Eyal, 1985; Ron et al., 1990), the Mojave desert California (Garfunkel, 1974; Schermer et al., 1996), the Sierran microplate, SE California (Lewis et al., 2007) and the Sumatra along strike variation has been explained as a plate locking and rigid rotation (McCaffrey et al., 2002; Prawirodirdjo et al., 1997). Block rotation seems to be related to lateral displacement of material parallel to master faults and out of the bulk shear zone (Schreurs, 1994).

The deformation produced by the rotation of kilometer-scale blocks in zones of distributed brittle deformation in the crust has been modeled combining different types of fault blocks models with the seismic moment tensor (Garfunkel, 1974; Jackson \&
McKenzie, 1988; Kissel \& Laj, 2012; Luyendyk et al., 1980; McKenzie \& Jackson, 1986; Ron et al. 1984; Wells \& Heller, 1988; Wernicke \& Burchfiel, 1982). However, no consensus has been found today whether the correct seismic moment tensor to use must be symmetric or asymmetric. Standard bibliography supports the postulate that the seismic moment tensor must be symmetric (Aki \& Richards, 2002; Backus \& Mulcahy, 1976a; Dahlen \& Tromp, 1998; Jackson \& McKenzie, 1988; Slawinski, 2010) and several authors support that it must be asymmetric (Abreu et al., 2018; Chapman \& Leaney, 2019; Molnar, 1983; Molnar \& Qidong, 1984; Twiss et al., 1993), with both versions (symmetric and asymmetric) completely supported by observations. However, without any doubt, the most routinely used version is the symmetric one.

Strictly speaking, the symmetric moment tensor contains less information compared to the asymmetric one. However, Jackson and McKenzie (1988) claims that this extra information is not relevant and cannot be observed from seismological observations. On the contrary, Molnar (1983) and Twiss et al. (1993) support that this extra information, contained in the asymmetric part, establishes the link between block rotations and focal mechanism solutions of seismic events. In particular, Twiss et al. (1993) establishes this connection using micropolar theory (Nagahama \& Teisseyre, 2000; Teisseyre, 1973, 2008, 2011; Teisseyre et al., 2006, 2008). This allows to model the discontinuous nature of faulting of distributed brittle deformation as a continuum, provided that the dimensions of the deforming material are large relative to the characteristic spacing of the discontinuities. In return, one can determine the characteristics of the regional deformation from an analysis of local discontinuous deformations characterized by faulting (block rotations) and by seismic activity.

Four main different versions of the asymmetric moment tensor have been proposed by Abreu et al. (2018), Molnar (1983), Twiss et al. (1993) and Chapman and Leaney (2019). These different versions seem to be different by definition and, in principle, contain different information. Here we show, however, that they are particular cases of a general alternative definition that can be postulated 
using micropolar theory. Because this alternative definition is based on micropolar theory, we propose a methodology to compute the new required parameters. We then apply the new model to an example of crustal block rotation produced by the Kaikōura earthquake occurred on the 14th of November 2016 in northeastern South Island. We run numerical simulations to compute seismograms using the opensource package SPECFEM3D GLOBE (Komatitsch \& Tromp, 1999) for the Kaikōura asymmetric moment tensor. We are able to predict observable differences produced by the new model which can serve as basis for future inversion studies. We finally discuss the contributions and further perspectives of this work.

\section{The Symmetric Seismic Moment Tensor}

We first recall basic concepts of the seismic moment tensor needed to present our theoretical developments. The seismic source is considered to be a localized failure in Hooke's law within a certain region of the space. The difference between the stress model that satisfies the constitutive Hooke's law $\tau^{\text {Hooke }}$ and the real (observed or true) stress $\tau^{\text {observed }}$ is called "the stress glut" $\tau^{\text {glut }}$ or "stress excess" (Backus \& Mulcahy, 1976a, b; Chapman, 2010; Dahlen \& Tromp, 1998). Hence we can write the following

$$
\tau^{\text {glut }}=\tau^{\text {Hooke }}-\tau^{\text {observed }} .
$$

The stress glut $\tau^{\text {glut }}$ has nonzero values in an explosion or in the fault zone of an earthquake (Chapman, 2010). For a small source, in terms of the wavelength and propagation distance, it is convenient to consider the volume average of the stress glut change in the source, i.e., the volume and time integral of the rate of change of stress glut over the support of the source (Chapman, 2010)

$$
M=\int_{t} \int_{V} \frac{\partial \tau^{\text {glut }}}{\partial t} d V d t=\int_{V}\left[\tau^{\text {glut }}\right] d V
$$

where $\left[\tau^{\text {glut }}\right]$ is the temporal jump of the stress glut and $M$ is known as the seismic moment tensor, with units of force times distance. We may write

$$
\tau_{i j}^{\text {glut }}=\overline{\mathbb{C}}_{i j k l} \varepsilon_{k l}^{P},
$$

where $\overline{\mathbb{C}}_{i j k l}$ are the average elastic parameters within the source region and $\varepsilon_{k l}^{P}$ is called the inelastic strain tensor in the faulting region, also called transformational strain (Ben-Zion, 2003).

Using distribution theory, where the term distribution refers to continuous linear functional on a space of smooth test functions, we can replace the observed or physical stress $\tau^{\text {observed }}$, which is in general discontinuous across the fault, by an associated regular distribution $(\mathcal{D})$ given by the following expression (Dahlen \& Tromp, 1998)

$$
\mathcal{D}\left(\tau^{\text {observed }}\right)=\overline{\mathbb{C}}_{i j k l} \mathcal{D}\left(\varepsilon_{i j}\right)=\frac{1}{2} \overline{\mathbb{C}}_{i j k l} \mathcal{D}\left(\partial_{i} u_{j}+\partial_{j} u_{i}\right) .
$$

The model (Hooke) stress $\tau^{\text {Hooke }}$ is, on the other hand, a singular distribution, given by the following expression (Dahlen \& Tromp, 1998)

$$
\tau^{\text {Hooke }}=\frac{1}{2} \overline{\mathbb{C}}_{i j k l}\left(\partial_{i} \mathcal{D} u_{j}+\partial_{j} \mathcal{D} u_{i}\right) .
$$

We therefore can write the stress glut $\tau^{\text {glut }}$ as follows (see Dahlen and Tromp (1998) Chapter 5 for further details)

$$
\begin{aligned}
\tau_{i j}^{\text {glut }} & =\frac{1}{2} \overline{\mathbb{C}}_{i j k l}\left[\left(\partial_{i} \mathcal{D} u_{j}+\partial_{j} \mathcal{D} u_{i}\right)-\mathcal{D}\left(\partial_{i} u_{j}+\partial_{j} u_{i}\right)\right] \\
& =\frac{1}{2} \overline{\mathbb{C}}_{i j k l} \Delta u_{k} n_{l},
\end{aligned}
$$

where $\Delta u$ is a displacement discontinuity vector across a surface $S$ with a unit normal $n$. We may write the seismic moment tensor Eq. (2) as follows

$$
M_{i j}(t)=\int_{V} \tau_{i j}^{\text {glut }} d V=\overline{\mathbb{C}}_{i j k l} P_{k l}(t),
$$

where $P(t)$ is called the seismic potency tensor (BenZion, 2003) given by the following expression

$$
P_{i j}(t)=\frac{1}{2} \int_{S} \Delta u_{i} n_{j} d S .
$$

For an isotropic material we can write the seismic moment as follows (Ben-Zion, 2003) 


$$
M_{i j}(t)=\int_{S}\left[\bar{\lambda} \delta_{i j} \Delta u_{k} n_{k}+\bar{\mu}\left(\Delta u_{i} n_{j}+\Delta u_{j} n_{i}\right)\right] d S .
$$

The first term on the right hand side of Eq. (9) is the scalar product of $\Delta u$ and $n$, which is zero when the two vectors are perpendicular to each other. This situation arises when $\Delta u$ is on the fault surface (Pujol, 2003), or for shear sources $(\Delta u \perp n)$ (Vavryčuk, 2005), for which we can write the following

$$
M_{i j}(t)=\int_{S} \bar{\mu}\left(\Delta u_{i} n_{j}+\Delta u_{j} n_{i}\right) d S .
$$

The scalar seismic potency of shear faulting on a planar surface is the integral of slip over the rupture area or, equivalently, the product of the spatial average of the final slip distribution and the failure area $A$ (Ben-Zion, 2003)

$$
P_{0}=\Delta u A,
$$

and the scalar seismic moment is (Chapman, 2010)

$$
M_{0}=\bar{\mu} \Delta u A,
$$

where $\bar{\mu}$ is the effective shear modulus (rigidity) in the source area.

The moment tensor defined in Eq. (7) is symmetric. This is a consequence of the conservation of mass and the balance of linear momentum together with the balance of angular momentum under the assumption of only central forces acting within the continuum, which requires that $\tau^{\text {Hooke }}$ and the elastic tensor $\mathbb{C}_{i j k l}$ are symmetric (Slawinski, 2010). As a consequence, the stress glut $\tau^{\text {glut }}$ is also symmetric.

Considering the observed stress $\tau^{\text {observed }}$ as the stress that satisfies the equation of motion, we can write the linear conventional elastic equation of motion as follows

$$
\rho \mathrm{\partial}_{t}^{2} u_{i}=\tau_{j i, j}^{\text {Hooke }}-\tau_{j i, j}^{\text {glut }},
$$

thus it becomes evident that the term $\tau_{j i, j}^{\text {glut }}$ behaves as a source of elastic motion. As noted by Chapman and Leaney (2019), it also becomes evident that the term $\tau^{\text {glut }}$ must be symmetric only due to the symmetries of the elastic tensor $\mathbb{C}_{i j k l}$ but it is not strictly required to be.

Inelastic deformation within a volume, where many earthquakes occur, can be described using seismic moment tensors: consider a volume $\Delta V$, during a time interval $\Delta t$, in which a large number $N$ of earthquakes occur with fracture areas $\Sigma_{x}$ and seismic moments $M_{i j}^{(x)}$. Kostrov (1974) has shown that the deformation can be written as follows

$$
\partial_{j} u_{i}=\frac{1}{\Delta V} \sum_{x=1}^{N} \int_{\Sigma^{x}} \Delta u_{i}^{(x)} n_{j}^{(x)} d S^{(x)}
$$

where $\Delta u^{(x)}$ is the slip for the earthquake number $x, n$ is the unit normal to the fault and $\Sigma^{x}$ is the surface area of the fault.

The increment of the mean deformation $\bar{\varepsilon}$ and mean vorticity $\overline{\mathrm{w}}$ in the volume $\Delta V$ during the time $\Delta t$ is given by the following expressions

$$
\bar{\varepsilon}_{i j}=\frac{1}{2}\left(\partial_{i} u_{j}+\partial_{j} u_{i}\right), \quad \overline{\mathrm{W}}_{i j}=\frac{1}{2}\left(\partial_{i} u_{j}-\partial_{j} u_{i}\right) .
$$

In virtue of Eq. (14), we can write the inelastic deformation as follows (Kostrov, 1974)

$$
\bar{\varepsilon}_{i j}^{\text {Kostrov }}=\frac{1}{2 \Delta V} \sum_{x=1}^{N} \int_{\Sigma^{x}}\left(\Delta u_{i}^{(x)} n_{j}^{(x)}+\Delta u_{j}^{(x)} n_{i}^{(x)}\right) d S^{(x)},
$$

which can be written in terms of the seismic moment tensor as follows

$$
\bar{\varepsilon}_{i j}^{\text {Kostrov }}=\frac{1}{2 \mu \Delta V} \sum_{x=1}^{N}\left(M_{i j}\right)^{(x)} .
$$

Equation (17) has been first found by Kostrov (1974) and subsequently used in several studies of inelastic deformation (Jackson \& McKenzie, 1988; Lewis et al., 2007; Matsumoto et al. 2016; Molnar \& Qidong, 1984; Twiss \& Unruh, 2007; Unruh et al., 1996). Equation (17) assumes that the distribution of inelastic strain in the volume is homogeneous after the occurrence of earthquakes for the target period. The homogeneous distribution of inelastic strain is equivalent to the homogeneous moment density in the volume. Thus, the assumption of homogeneous strain in the volume is only appropriate if many earthquakes occur in the volume. Therefore, the spatial distribution of the moment tensor needs to be considered when evaluating the applicability of this method (Matsumoto et al., 2016). 


\section{What Can an Asymmetric Moment Tensor Explain that a Symmetric One Cannot?}

The symmetric moment tensor given by Eq. (10) is routinely used nowadays to describe seismic sources. It has, however, some limitations: Molnar (1983) introduced an asymmetric moment tensor motivated by observations in the Haiyuan fault in the Ningxia-Hui Shilong region of China and the observed surface deformation of several large earthquakes in eastern China. He showed a case of simple shear where the expected moment tensor happens to be asymmetric.

A symmetric seismic moment tensor as described by Eq. (10) has been the pillar for the fundamental physical description of the earthquake rupture in the last decades. However, Molnar (1983) has shown simple cases where the symmetric moment tensor fails to describe the rotation that occurs during simple shear. Figure 1 shows a simple example where the faults intersect the edge of the region under consideration. Slip occurs on parallel faults so that the region under consideration undergoes simple finite shear $\left(\varepsilon_{x x}=\varepsilon_{y y}=\varepsilon_{y x}=0, \varepsilon_{x y} \neq 0\right)$. If a symmetric strain tensor is used to describe the moment tensor (Eq. 9), then each event is assumed to contribute equally to $\varepsilon_{y x}$ and $\varepsilon_{x y}$, so that pure shear develops instead of simple shear (Molnar, 1983). The symmetric moment tensor predicts the correct amount of average strain in the region but it neglects the rotation $\left(=1 / 2 \partial u_{x} / \partial y\right.$ in this case) that occurs during simple shear. The observed strain deformation tensor $\varepsilon_{i j}$ can be easily computed and related to a seismic moment tensor using Eq. (17). The average strain is given by the following expression (see Allmendinger et al. (1989) for more details)

$$
\begin{aligned}
\bar{\varepsilon}_{i j} & =\frac{M_{0}}{\mu V}\left[\begin{array}{ccc}
-\sin \phi \cos \phi & \sin ^{2} \phi & 0 \\
-\cos ^{2} \phi & \sin \phi \cos \phi & 0 \\
0 & 0 & 1
\end{array}\right] \\
& =M_{0} \Delta u_{i} n_{j}=\frac{M_{i j}^{\text {Molnar }}}{2 \mu V} .
\end{aligned}
$$

Clearly the strain induced deformation is asymmetric. In virtue of Eq. (18), the moment tensor $M_{i j}^{\text {Molnar }}$ is also asymmetric.
Therefore, Molnar (1983) makes the assumption that it is generally possible to distinguish the fault and the auxiliary planes in deforming zones (Jackson \& McKenzie, 1988), which allows him to define an asymmetric moment tensor as follows

$$
M_{i j}^{\mathrm{Molnar}}=M_{0} \Delta u_{i} n_{j},
$$

where $\Delta u_{i}$ is a unit vector parallel to the direction of the slip and $n_{j}$ is the unit normal parallel to the fault plane. A moment tensor like Eq. (19) is much more useful compared to Eq. (9) since it defines nine independent components to describe the physics of the earthquake rupture instead of six. However, Molnar's definition has been criticized because it does not agree with the conservation of angular momentum which requires that $\sigma^{\text {Hooke }}$ and the elastic tensor $\mathbb{C}_{i j k l}$ are symmetric (Jackson \& McKenzie, 1988; Slawinski, 2010).

Using simple analytical examples, Jackson and McKenzie (1988) have shown that the antisymmetric part of the seismic moment tensor defined by Molnar (1983) (Eq. 19), measures only the local rotation of the medium and therefore it cannot be recorded in seismic data. Arriving at the same conclusion, Twiss et al. (1993) and Twiss (2009) have shown that the anti-symmetric part of Molnar's moment tensor Eq. (19) is a non-objective variable and thus it cannot be measured by seismic data. The objective variables that appear in constitutive equations define the intrinsic characteristics of the deformation, whereas non-objective variables do not. For instance, the macroscopic deformation rate defined as follows

$$
\mathrm{w}_{k i}=\frac{1}{2}\left(\partial_{k} v_{i}-\partial_{i} v_{k}\right), \quad v_{i}=\partial_{t} u_{i},
$$

is a non-objective variable because the magnitude depends on the coordinate system in which it is measured (Eringen, 1980; Twiss et al., 1993).

A problem arises because using seismic data and geological constrains, Molnar and Qidong (1984) have calculated components of a global asymmetric seismic moment tensor for sets of earthquakes in different tectonic provinces in central eastern Asia. Thus, in contrast to the arguments of Jackson and McKenzie (1988), the results obtained by Molnar and Qidong (1984) suggest that seismic moment tensors can be asymmetric and observed in seismic data. 
(a)

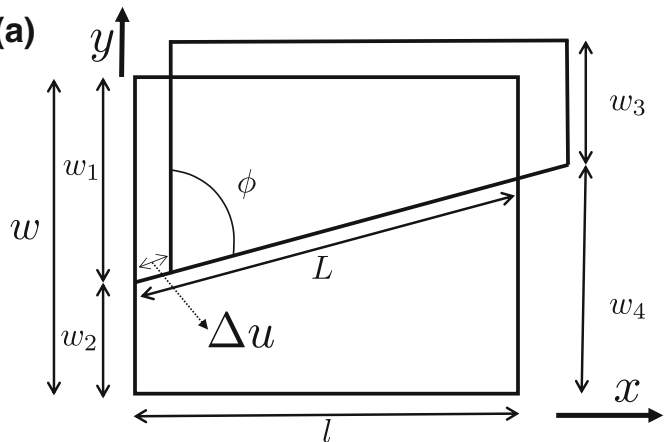

(c)

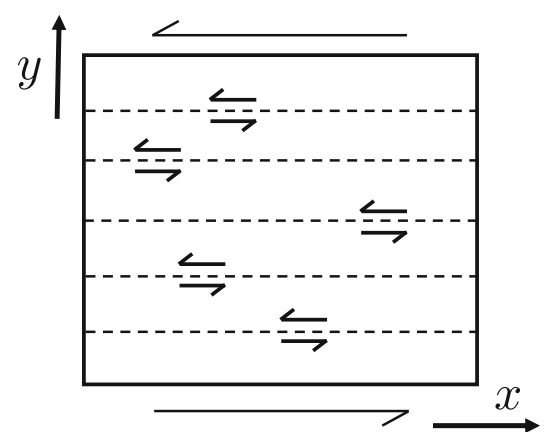

(b)

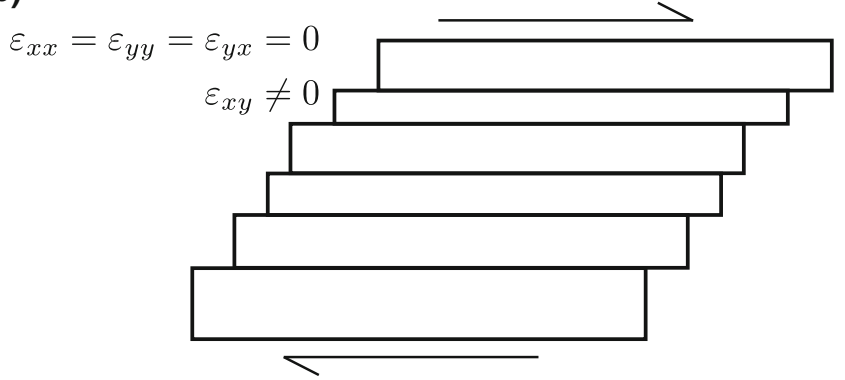

(d)

$$
\begin{aligned}
& \varepsilon_{x x}=\varepsilon_{y y}=0 \\
& \varepsilon_{x y}=\varepsilon_{y x} \neq 0
\end{aligned}
$$

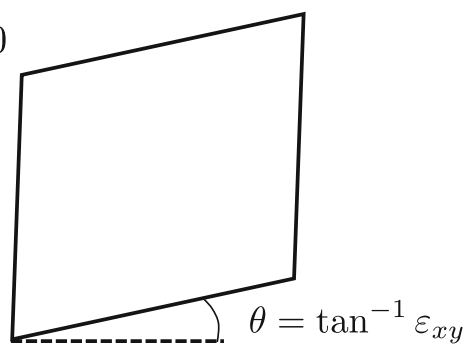

Figure 1

a rectangular parallelepiped cut by a vertical fault with strike $\phi$. The dimensions of the body are $l, w$ and thickness $h$. Slip displacement $\Delta u$ occurs on a fault of length $L=l / \sin \phi$. The slip displacement $\Delta u$ is small compared with $l, w$ and $L$. The width $w$ is cut into segments of length $w_{1}$ and $w_{2}$ on the left and $w_{3}$ and $w_{4}$ on the right. b-d illustration of a case where the assumption of a symmetric moment tensor does not predict the correct strain field. b Series of faults parallel to the $x$ axis, slip by right-lateral shear. c As a result, the average finite strain is simple shear with $\varepsilon_{x y}=\partial u_{x} / \partial y$ finite and $\varepsilon_{x x}=\varepsilon_{y y}=\varepsilon_{y x}=0$. d If a symmetric stress tensor is used, then the irrotational strains $\varepsilon_{x y}$ and $\varepsilon_{y x}$ must be equal so that the resulting strain field must be pure shear. Note that this assumption neglects the rotation that occurs in simple shear. After Molnar (1983)

These results motivated Twiss et al. (1993) to state that classical continuum theory cannot account for all observable characteristic of the deformation and a more general expression of the seismic moment tensor is needed.

Trying to be consistent with the arguments of Jackson and McKenzie (1988), Eringen (1980) and still keeping an asymmetric moment tensor in order to include the field observations of Molnar and Qidong (1984) and Twiss et al. (1993) stated that Molnar's moment tensor (Eq. 19) can be kept with a slight modification: the slip direction $\Delta u_{i}$ defined in Molnar's moment tensor must be changed. Twiss et al. (1993) defines a local micropolar seismic moment tensor for an earthquake $M_{i j}^{\text {Twiss }}$ and a global micropolar seismic moment tensor $\bar{M}_{i j}^{\text {Twiss }}$ as follows

$$
M_{i j}^{\text {Twiss }}=M_{0} \Delta u_{i}^{\text {Twiss }} n_{j}, \quad \bar{M}_{i j}^{\text {Twiss }}=\sum_{\alpha=1}^{N} M_{i j}^{\text {Twiss }},
$$

where $M_{0}$ is the scalar seismic moment and the summation is over $N$ earthquakes occurring in a given volume and $\Delta u_{i}^{\text {Twiss }}$ is the slip vector given by micropolar theory instead of classical continuum theory. Note that $M_{i j}^{\mathrm{Twiss}}$ is similar to $M_{i j}^{\mathrm{Molnar}}$ asymmetric moment tensor with the difference in the definition of the slip direction only.

Twiss et al. (1991, 1993) found that the slip direction, or the direction of maximum macroshearing on a micro-material plane (observed direction of the shearing), in micropolar media is given by the following expression 


$$
\Delta u_{i}^{T \text { wiss }}=\frac{1}{L}\left[\left(d_{k i}+\frac{1}{2} \epsilon_{k i j} \Psi_{j}\right) \eta_{k}-d_{k l} \eta_{k} \eta_{l} \eta_{i}\right],
$$

where $L$ is the magnitude of the vector between brackets, $\Psi$ the net vorticity and

$$
d_{k i}=\frac{1}{2}\left(\partial_{k} u_{i}+\partial_{i} u_{k}\right),
$$

where $\eta_{k}$ is the unit normal to a micro-material plane that is part of the boundary of a block. The instantaneous direction of displacement on a shear plane is necessarily the direction of maximum rate of shear on that plane.

Twiss et al. (1991, 1993) argue that the micropolar theory resolves the symmetry problem of the asymmetric moment tensor defined by Molnar (1983) and, at the same time, obeys the law of conservation of angular momentum, allowing to be used with seismic data. Although the theory is formulated in terms of rates of deformation (Eq. 23), in practice, Twiss et al. (1991, 1993) interpret these rates to be represented by small increments of deformation that accumulate over a finite but geologically very short time interval $\Delta t$. Elimination of time from the rate terms by multiplying each term by $\Delta t$ does not affect the results. Thus, the rates are represented by the instantaneous deformation, and the time interval over which these increments accumulate is not a factor in the analysis (Twiss and Unruh 2007).

There are however, some remaining inconvenients with the moment tensor defined by Twiss et al. (1991, 1993) in Eq. (21). One inconvenient is related to the elastic material parameters defined in the stress glut Eq. (3). In micropolar elasticity, the tensor of elastic parameters $\mathbb{C}_{i j k l}$ is non-symmetric, thus at least one new elastic constant is introduced, the Cosserat couple modulus $\mu_{c}$ in isotropic media, but it is not taken into account in the definition of Eq. (21). Second, for finding the asymmetric moment tensor one needs to be able to invert for the slip direction Eq. (22), which is related to the deformation strain rate Eq. (23), which is usually measured using several seismic events.

Woodhouse (1981) pointed out that if a fault crossed a material discontinuity, determining the moment tensor was ambiguous and the fraction of the fault on each side of the discontinuity must be specified a priori. The word ambiguous, as explained by Chapman and Leaney (2019), refers to the fact that the value of the moment tensor depends on the exact location of the source. As such, it does not provide a useful parameterization for a source near a discontinuity. For the ambiguous parameters, the source location must be specified exactly and not just as being "at" the discontinuity. Because the moment tensor is discontinuous across the interface for slip parallel to the discontinuity, it is ambiguous even though it has a well-defined intermediate value for slip on the discontinuity (Chapman \& Leaney, 2019).

In order to include torque in the seismic moment tensor and to be consistent with Molnar's asymmetric moment tensor, Chapman and Leaney (2019) have explicitly included the antisymmetric part in the definition of the moment tensor Eq. (7) as follows

$$
M_{i j}^{\text {Chapman }}=\overline{\mathbb{C}}_{i j k l} P_{k l}+M_{i j}^{A},
$$

where $M_{i j}^{A}$ is the antisymmetric part of $M_{i j}$ and $P_{k l}$ the potency density tensor defined in Eq. (8). This allows Chapman and Leaney (2019) to show that for a general source and any location "at" an interface, certain components of its moment tensor and certain components of its potency tensor can be determined unambiguously. However, the definition proposed by Chapman and Leaney (2019) is ad hoc and does not have any physical origin.

In the next section we reconcile Molnar (1983), Jackson and McKenzie (1988), Twiss et al. (1993) and Chapman and Leaney (2019) points of view by introducing an alternative asymmetric moment tensor.

\section{The Micropolar Moment Tensor: An Application to the Kaikoura Event}

\subsection{The Asymmetric Mircopoplar Moment Tensor}

Let us now define an asymmetric moment tensor using the concepts of stress-glut in micropolar media and distribution theory presented in the previous section. For doing so, we use reduced micropolar model (Grekova, 2012a, b, 2016; Grekova et al., 2009; Kulesh et al., 2009). In such a medium, body points have independent translational and rotational 
degrees of freedom, but no stresses are induced by the gradient of microrotation. We further discuss this choice at the end of the section. Let first recall the linear elastic equations of motion for a reduced micropolar media

$$
\begin{aligned}
\rho \partial_{t}^{2} u_{j} & =\partial_{i} \sigma_{i j}, \quad \text { balance of linear momentum } \\
I \partial_{t}^{2} \theta_{j} & =\epsilon_{j k l} \sigma_{k l}, \quad \text { balance of angular momentum }
\end{aligned}
$$

where $\sigma$ is the asymmetric micropolar stress tensor, $\epsilon$ is the Levi-Civita symbol and $\theta$ is the independent rotation vector. The free surface boundary conditions are given by

$$
\hat{n} \cdot \sigma=0 \quad \text { on } \quad \partial \Omega,
$$

where $\hat{n}$ refers to the direction normal to the surface $\partial \Omega$. In an isotropic and homogeneous media, the stress $\sigma_{i j}$ and strain $\epsilon_{i j}$ tensors are given by

$$
\begin{gathered}
\sigma_{i j}=\mu\left(\partial_{i} u_{j}+\partial_{j} u_{i}\right)+\lambda \delta_{i j} \partial_{k} u_{k}+2 \mu_{c} \epsilon_{i j k} \Psi_{k}, \\
e_{i j}=\frac{1}{2}\left(\partial_{i} u_{j}+\partial_{j} u_{i}\right)+\epsilon_{i j k} \Psi_{k},
\end{gathered}
$$

where $\mu_{c}$ is the Cosserat couple modulus, a new elastic parameter measuring the ability of the material to be deformed by rotational motions, $\Psi$ is the effective rotational motion or net vorticity vector (Twiss et al., 1993) defined as the difference between the curl $\left(\frac{1}{2} \epsilon_{k a b} \partial_{a} u_{b}\right)$ and the independent rotation $\theta$ as follows

$$
\Psi_{k}=\left(\frac{1}{2} \epsilon_{k a b} \partial_{a} u_{b}-\theta_{k}\right) .
$$

Using Eq. (1), we can write the following reduced micropolar linear equations (Abreu et al., 2018)

$$
\begin{aligned}
\rho \partial_{t}^{2} u_{i} & =\sigma_{j i, j}-\sigma_{j i, j}^{\text {glut }} \\
I \partial_{t}^{2} \theta_{i} & =\epsilon_{i j k} \sigma_{j k}-\epsilon_{i j k} \sigma_{j k}^{\text {glut }} .
\end{aligned}
$$

Using distribution theory (see Dahlen and Tromp (1998) Chapter 5 for further details), it is straightforward to show that in micropolar media, the stress glut $\sigma^{\text {glut }}$ is given by the following expression

$$
\begin{aligned}
\sigma_{i j}^{\text {glut }} & =\frac{1}{2} \overline{\mathbb{C}}_{i j k l}\left(\partial_{i} \mathcal{D} u_{j}+\partial_{j} \mathcal{D} u_{i}\right) \\
& +\epsilon_{i j k} \mathcal{D} \Psi_{k}-\mathcal{D}\left(\frac{1}{2}\left(\partial_{i} u_{j}+\partial_{j} u_{i}\right)+\epsilon_{i j k} \Psi_{k}\right) \\
& =\overline{\mathbb{C}}_{i j k l} \Delta u_{k}^{\text {Twiss }} n_{l},
\end{aligned}
$$

where $\Delta u^{\text {Twiss }}$ is a displacement discontinuity vector across a surface $S$, defined by Eq. (22), with a unit normal $n$ to the fault plane. We can define the micropolar seismic moment tensor as follows

$$
M_{i j}^{\text {micropolar }}(t)=\int_{V} \sigma_{i j}^{\text {glut }} d V
$$

and assuming isotropy in the elastic parameters we may write

$$
\begin{aligned}
& M_{i j}^{\text {micropolar }}(t)=\frac{1}{2} \int_{S}\left[\lambda \delta_{i j} \Delta u_{k}^{\text {Twiss }} n_{k}\right. \\
& +\bar{\mu}\left(\Delta u_{i}^{\text {Twiss }} n_{j}+\Delta u_{j}^{\text {Twiss }} n_{i}\right) \\
& \left.+\bar{\mu}_{c}\left(\Delta u_{i}^{\text {Twiss }} n_{j}-\Delta u_{j}^{\text {Twiss }} n_{i}\right)\right] d S .
\end{aligned}
$$

For shear sources $(\Delta u \perp n)$, Eq. (33) reduces to the following expression

$$
\begin{aligned}
& M_{i j}^{\text {micropolar }}(t)=\frac{1}{2} \int_{S}\left[\bar{\mu}\left(\Delta u_{i}^{\text {Twiss }} n_{j}+\Delta u_{j}^{\text {Twiss }} n_{i}\right)\right. \\
& \left.+\bar{\mu}_{c}\left(\Delta u_{i}^{\text {Twiss }} n_{j}-\Delta u_{j}^{\text {Twiss }} n_{i}\right)\right] d S .
\end{aligned}
$$

The similarities between the isotropic and Twiss's micropolar moment tensor become evident, since we can write Twiss's seismic moment tensor as follows

$$
\begin{aligned}
M_{i j}^{\text {Twiss }} & =M_{0} \Delta u_{i}^{\text {Twiss }} n_{j}=\bar{\mu} A \Delta u_{i}^{\text {Twiss }} n_{j} \\
& =\frac{1}{2} \int_{S}\left[\bar{\mu}\left(\Delta u_{i}^{\text {Twiss }} n_{j}+\Delta u_{j}^{\text {Twiss }} n_{i}\right)\right. \\
& \left.+\bar{\mu}\left(\Delta u_{i}^{\text {Twiss }} n_{j}-\Delta u_{j}^{\text {Twiss }} n_{i}\right)\right] d S,
\end{aligned}
$$

again, taking into account that the slip direction is given by Eq. (22). One can observe that Twiss's seismic moment tensor is a particular case of the micropolar moment tensor presented in Eq. (34). Note that for $\mu_{c}=\mu$ we have $M_{i j}^{\text {Twiss }}=M_{i j}^{\text {micropolar }}$ and for $\mu_{c} \rightarrow \infty$ we have $M_{i j}^{\text {micropolar }}=M_{i j}^{\text {symmetric }}$. Thus, 
depending on the selection of the Cosserat couple modulus $\mu_{c}$, micropolar theory can reconcile both symmetric and asymmetric points of view. Similarities between the isotropic micropolar and Chapman's (Eq. 24) moment tensors also become evident. Both models are equivalent if we write the following

$$
M_{i j}^{A}=\frac{1}{2} \int_{S} \bar{\mu}_{c}\left(\Delta u_{i} n_{j}-\Delta u_{j} n_{i}\right) d S .
$$

Equation (34) is valid in a reduced micropolar medium. Using a more generalized micropolar theory with a non zero couple stress tensor would have led to a moment tensor dependent on (local) slip rotational motions. We will develop the more generalized theory in future works. In the next section we show how to obtain the Cosserat couple modulus $\mu_{c}$ in a realistic scenario. This will lead to the realistic calculations of the introduced micropolar moment tensor.

\subsection{Computing the Cosserat Couple Modulus $\mu_{c}$ Using Homogenization of a Multilayered Periodic Medium}

Micropolar theory can be used to model the behavior of granular, layered and blocky rock media (Mühlhaus, 1993). In the case of the layered material, the nonstandard terms of the micropolar theory can be used to model the influence of the bending stiffness of each layer on the material's response. In this way, a second (internal) length scale is introduced in addition to the global one, defined for example by structural dimensions. In this specific case, the internal lengths are simply the layer thicknesses (see Fig. 2).

Biot (1965) showed that, within certain limits, a laminated periodic medium behaves like a classical elastic continuum with anisotropic properties, although the individual layers may be isotropic. The validity of this approximation is restricted to cases where the rigidity contrasts between the layers are not too large and the layer thicknesses remain sufficiently small with respect to the dominant wavelength in the deformation field (layer thickness). Similar approximations have also been proposed in the geophysical field by Backus (1962) with the same kind of limitations. Strictly speaking, a layered material can be modeled as a conventional orthotropic continuum only if the layer thickness is negligible compared with a characteristic structural length (Backus, 1962; Mühlhaus, 1993).

In order to overcome this long time standing limitations, Mühlhaus and Vardoulakis (1986) and Mühlhaus (1990) proposed an alternative approach by representing an incompressible laminated halfspace as a homogeneous half-space of Cosserat (micropolar) material (Papamichos et al., 1990). The internal length, introduced by the micropolar's theory, provides the way to include moment stresses in the constitutive model and to remove the assumption that the layers are small compared with the characteristic structural length (Mühlhaus, 1990, 1993; Mühlhaus \& Triantafyllidis, 1987; Mühlhaus \& Vardoulakis, 1986; Papamichos et al., 1990; Sulem \& Vardoulakis, 1995). The micropolar continuum theory permits geometric properties of (a)

anisotropic linear elastic model

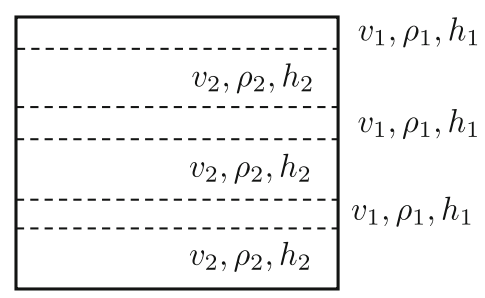

(b) simple shear forces applied

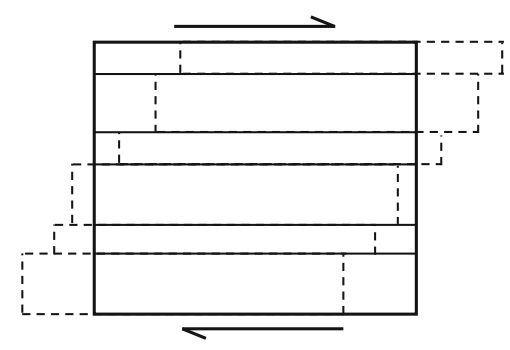

Figure 2 (c)

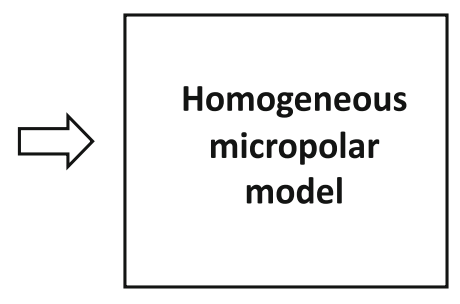

a Periodic laminated medium with two thickness $h_{1}, h_{2}\left(h_{2}>h_{1}\right)$. The number of layer is infinite. b Simple shear applied in the direction of the layering. c Homogeneous micropolar equivalent model 
certain material inhomogeneities to be considered only in the constitutive relationships.

The elastic parameters of the micropolar continuum are identified by stipulating the incremental work in the respective deformations in the micropolar and the classical continuum to be equal. In this case, the Cosserat couple modulus $\mu_{c}$ can be written as follows (see Mühlhaus and Vardoulakis (1986) and Mühlhaus (1990) for further details)

$$
\mu_{c}=\mu^{V}-\mu^{R},
$$

where $\mu^{V}, \mu^{R}$ refer to the Voigt's modulus and Reuss's shear modulus respectively given by the following expressions

$$
\mu^{V}=x_{1} \mu_{1}+x_{2} \mu_{2}, \quad \mu^{R}=\frac{1}{\frac{x_{1}}{\mu_{1}}+\frac{x_{2}}{\mu_{2}}}=\frac{\mu_{1} \mu_{2}}{x_{2} \mu_{1}+x_{1} \mu_{2}} .
$$

where $x_{1}, x_{2}$ are the volume fractions of the two materials (see Fig. 2) (Mühlhaus, 1993; Papamichos et al., 1990). The equivalent shear modulus of the layered medium is taken to be

$$
\mu^{\text {effective }}=\mu^{R} .
$$

The Cosserat couple modulus defined by Mühlhaus and Vardoulakis (1986) and Mühlhaus (1990) as the difference between Voigt and Reuss moduli in Eq. (37) is related to the magnitude of the elastic anisotropy (Chung \& Buessem, 1967; Hill, 1952).
To gain some feeling on the values that the Cosserat couple modulus $\mu_{c}$ can adopt, we use Mühlhaus and Vardoulakis (1986) calculations and we first show on Fig. 3a that the Voigt and the Reuss averages provide the upper and lower bound of the elastic modulus of a composite material, in general, the real shear modulus of the composite lies between the two curves (Watt et al., 1976). In particular, the Voigt model is related to axial loading of the composite and the Reuss model is related to transverse loading of the composite. We use $\mu_{2} / \mu_{1}=0.5$ for the calculations of Fig. 3a.

We also show in Fig. $3 b$ the predicted values of the Cosserat couple modulus normalized by the values of the shear modulus in layer type $1, \mu_{c} / \mu_{1}$ using Eq. (37), as a function of the material fraction $x_{1}$ and of the material properties $\mu_{2} / \mu_{1}$. Figure $3 \mathrm{~b}$ shows that large $\mu_{c} / \mu_{1}$ occur for small $\mu_{2} / \mu_{1}$ and large volume fractions $x_{1}$, and that, $\mu_{c}$ is always smaller than the shear modulus $\mu_{1}$. We do not expect the shear modulus ratio $\mu_{2} / \mu_{1}$ to be lower than 0.5 at most, meaning that $\mu_{c} / \mu_{1}$ is likely lower than 0.1 .

We can additionally relate the homogenized micropolar model to the classical transverse isotropic model. Postma (1955) showed that a periodic structure consisting of alternating plane, parallel, isotropic, and homogeneous elastic layers can be replaced by a homogeneous, transversely isotropic material as far as its gross-scale elastic behavior is concerned (see Fig. 4). As previously mentioned, Biot (1965) also showed that, if the layer thickness is (a)

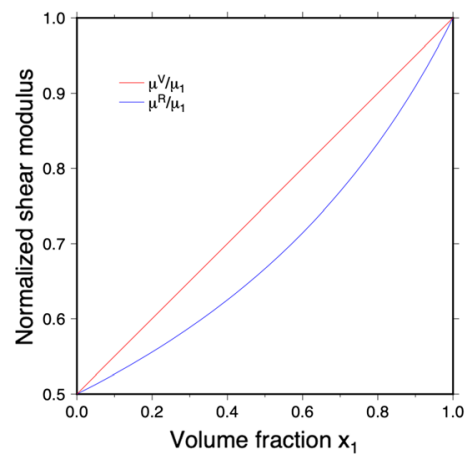

(b)

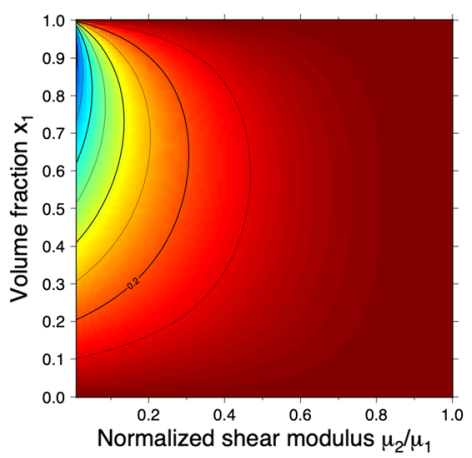

(c)

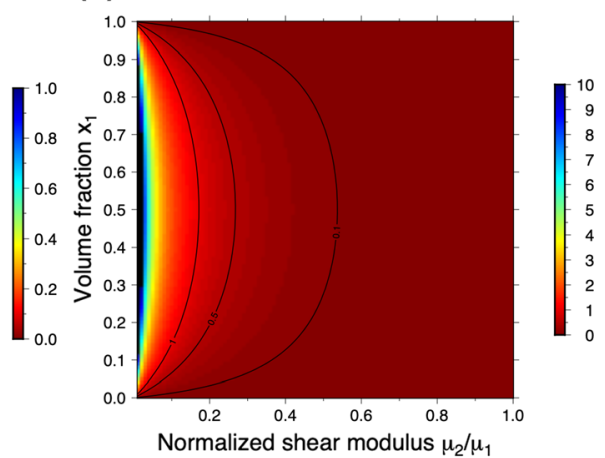

Figure 3

a Voigt and Reuss averages as a function of the volume fraction $x_{1}$ for $\mu_{2} / \mu_{1}=0.5$. b Values of the Cosserat couple modulus $\mu_{c}$ normalized by the shear modulus $\mu_{1}$ computed using Eq. (37) and $\mathbf{c}$ values of the Cosserat couple modulus $\mu_{c}$ normalized by the Reuss shear modulus $\mu^{R}$ 
(a)

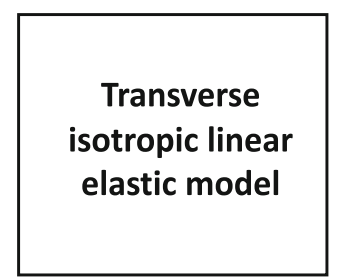

(b) Layered linear elastic model

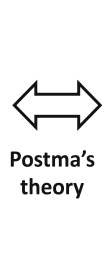

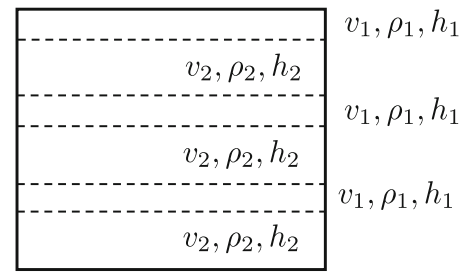

Figure 4 (c)

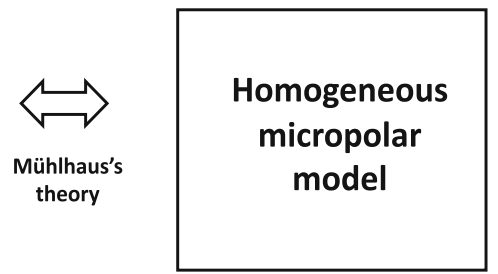

Equivalences between three different types of continuum models. Postma (1955) have shown the equivalence between a homogeneous transverse isotropic model and periodic structure consisting of two alternating plane, parallel, isotropic, and homogeneous elastic layers with certain density $\rho$, velocity $v$ and thickness $h$, which is in turn an equivalent micropolar models as shown by Mühlhaus (1993)

negligibly small compared with a characteristic structural length, a laminated periodic medium behaves like a classical elastic continuum with transverse isotropy. Similarly, Mühlhaus and Vardoulakis (1986) and Mühlhaus (1990, 1993) showed that the same layered periodic structure proposed by Postma (1955) can be replaced by a homogeneous micropolar model (see Fig. 4). The advantage of the micropolar model is that it does not assume that the layers (of layered periodic structure) are small compared with the characteristic structural length (Mühlhaus, 1990, 1993; Mühlhaus \& Triantafyllidis, 1987; Mühlhaus \& Vardoulakis, 1986; Papamichos et al., 1990; Sulem \& Vardoulakis, 1995).

\subsection{Application to the Kaikoura event, $14^{\text {th }}$ of November 2016, Mw 7.8}

The main motivation to apply micropolar theory in the description of brittle fracture deformation comes from the fact that continuum mechanics fails to describe the average motion of the shear planes that form the surfaces of the rotating blocks observed in the field. This is because those planes must rotate with the blocks rather than with the large-scale continuum motion. In this section we show how to compute the micropolar moment tensor presented in Sect. 4.

One important example of observed block rotation is in the North and South Islands of New Zealand (Ron et al., 1984; Roberts, 1995; Walcott, 1984; Wang et al., 2020). We select the Kaikōura event that occurred on the 14th of November 2016, northeastern
South Island with moment magnitude (Mw) 7.8. The Kaikōura earthquake was the most powerful event experienced in the region in more than 150 years (Hamling et al., 2017). Field observations, in conjunction with interferometric synthetic aperture radar (InSAR), Global Positioning System (GPS), and seismology data, reveal the Kaikoura earthquake to be one of the most complex earthquakes ever recorded with modern instrumental techniques (Hamling et al., 2017). Various studies show a clear pattern of clockwise block rotation bounded by three nearlyorthogonal surface ruptures produced by the Kaikōura earthquake (Hamling et al. 2017; Shi et al., 2019; Wang et al., 2020). Crustal block rotation played a significant role in releasing accumulated strain, possibly preventing further rupture propagation along the Hope fault (Wang et al., 2020). This suggests that coseismic rotation may help to accommodate plate boundary propagation.

The location of the Kaikōura earthquake and the fault involved in the rupture are shown in Fig. 5. To apply micropolar theory for the description of the block rotations we must compute the asymmetric micropolar moment tensor given in Eq. (34) using information of the symmetric moment tensor obtained by different seismological inversion techniques.

The standard symmetric moment tensor $M_{i j}^{\text {sym }}$ decomposition is given by the following expression (Udias \& Buforn, 2018)

$$
M_{i j}^{\mathrm{sym}}=M_{i j}^{\mathrm{ISO}}+M_{i j}^{\mathrm{DC}}+M_{i j}^{\mathrm{CLVD}}
$$

where $M^{\mathrm{ISO}}$ refers to the isotropic part (a change in 

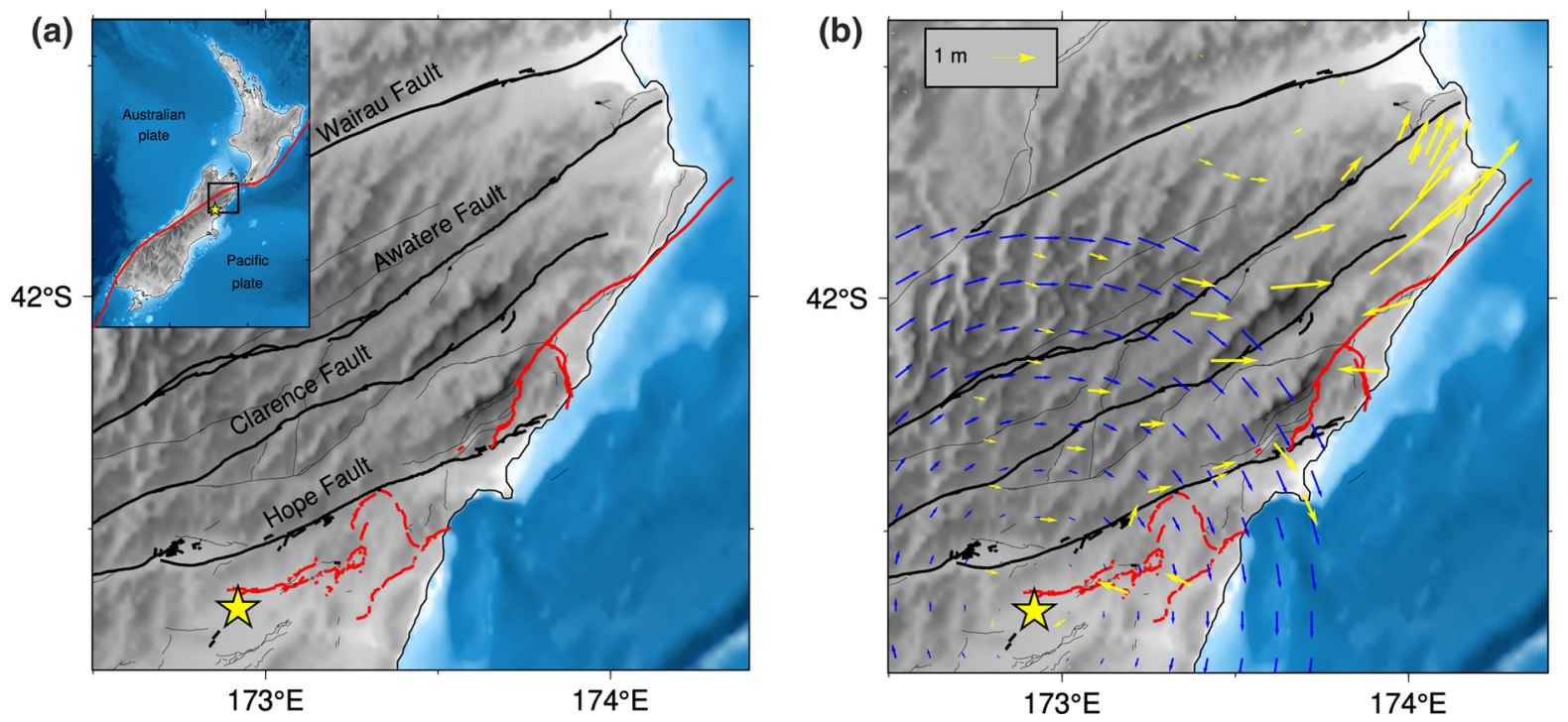

Figure 5

a Tectonic setting of the Marlborough Fault Zone (Langridge et al., 2016) showing all active faults (black lines) and the 2016 Kaikōura earthquake (yellow star) ruptures (red curves). b Rotational field $\overline{\mathbf{W}}_{i j}^{\text {Total }}$ (blue vectors) computed from $M^{\text {skew }}\left(\mu_{c} / \mu^{R}=0.5\right)$ compared to observed GPS coseismic displacements (yellow vectors) (Hamling et al., 2017). It is important to note that while GPS data are given in meters, we have not inverted for the amplitude of the rotational field $\bar{w}_{i j}^{\text {Total }}$. The purpose is to show that the direction of the rotational field obtained using micropolar theory is in agreement with GPS observations

volume like in an explosion), $M^{\mathrm{DC}}$ refers to the double-couple part and $M^{\mathrm{CLVD}}$ refers to the compensated linear vector dipole part. The physical interpretation of $M^{\mathrm{CLVD}}$ is a sudden change in the shear modulus in a direction normal to the fault plane, without changes in volume (Udias \& Buforn, 2018). The double-couple part can be written as follows [see Eq. (10)]

$$
M_{i j}^{\mathrm{DC}}=\frac{\bar{\mu} A}{2}\left(\Delta u_{i} n_{j}+\Delta u_{j} n_{i}\right),
$$

where $\Delta u$ is the slip vector and $n$ the unit normal to the fault plane and $A$ is the area of rupture. We can rewrite the micropolar moment tensor Eq. (34) in terms of the double-couple $M_{i j}^{\mathrm{DC}}$ as follows

$$
\begin{aligned}
M_{i j}^{\text {micropolar }} & =\frac{\bar{\mu} A}{2}\left[\left(\Delta u_{i} n_{j}+\Delta u_{j} n_{i}\right)+\frac{\mu_{c}}{\bar{\mu}}\left(\Delta u_{i} n_{j}-\Delta u_{j} n_{i}\right)\right], \\
& =M_{i j}^{\mathrm{DC}}+M_{i j}^{\mathrm{skew}},
\end{aligned}
$$

where we have called

$$
M_{i j}^{\text {skew }}=\frac{\mu^{R} A}{2}\left[\frac{\mu_{c}}{\mu^{R}}\left(\Delta u_{i} n_{j}-\Delta u_{j} n_{i}\right)\right],
$$

and we have replaced the average shear modulus $\bar{\mu}$ by the Reuss average $\mu^{R}$ (Eq. 38)). Equation (42) means that if we have information on the double-couple part of the moment tensor $M_{i j}^{\mathrm{DC}}$, obtained for example from the CMT Catalog (Ekström et al., 2012), and we compute the ratio $\mu_{c} / \mu^{R}$, we will have access to the micropolar moment tensor. Note that this is because we have access to the term $\mu^{R} A / 2$ directly from $M_{i j}^{\mathrm{DC}}$. The slip vector $\Delta u$ and the unit vector normal to the fault plane $n$ can be computed from the eigenvectors of the seismic moment tensor (Udias \& Buforn, 2018; Vavryčuk, 2005) as follows

$$
n=\frac{1}{\sqrt{2}}(p+t), \quad \Delta u=\frac{1}{\sqrt{2}}(p-t),
$$

where $p$ and $t$ are the unit eigenvectors of the moment tensor corresponding to the pressure $(\mathrm{P})$ and tension (T) axes.

We recall that the physical meaning of the Cosserat couple modulus $\mu_{c}$ is a measure of seismic anisotropy (see Sect. 4.2), thus the antisymmetric part 
of the seismic moment tensor defined in Eq. (43) is simply a contribution of a certain level of anisotropy to the seismic moment tensor, which allows to model coseismic block rotations.

We define the total asymmetric moment tensor $M_{i j}^{\text {Total }}$ given by combining symmetric (Eq. 40) and anti-symmetric (Eq. 42) parts as follows

$$
\begin{aligned}
M_{i j}^{\text {Total }} & =M_{i j}^{\mathrm{ISO}}+M_{i j}^{\mathrm{CLVD}}+M_{i j}^{\text {micropolar }}, \\
& =M_{i j}^{\mathrm{ISO}}+M_{i j}^{\mathrm{DC}}+M_{i j}^{\mathrm{CLVD}}+M_{i j}^{\mathrm{skew}}, \\
& =M_{i j}^{\mathrm{sym}}+M_{i j}^{\text {skew }} .
\end{aligned}
$$

Note, however, that we have assumed that the slip direction of the micropolar and symmetric moment tensors are the same. Twiss et al. (1991, 1993), Twiss and Unruh (2007), Twiss (2009) and Lewis et al. (2007) found the slip direction of micropolar theory (Eq. 22) in several studies by inverting a set of event data within a deforming area. This is, unfortunately, impractical from a seismological point of view since seismologists usually analyze, and invert for, seismic focal mechanisms of individual events. For simplification purposes, and for the aim of this study, we assume that the unit vectors of the slip $\Delta u$ and fault normal $n$, as well as the term $\mu^{R} A$ can be directly obtained from the individual seismic moment tensors found in the CMT catalog (Ekström et al., 2012). The only parameter left is thus $\mu_{c} / \mu^{R}$.

From Fig. $4 \mathrm{c}$, it seems that for reasonable values of $\mu_{2} / \mu_{1}$ we will always get $\mu_{c} / \mu^{R}$ lower than 1 . We emphasize, however, that our aim here is to find differences in waveforms predicted by linear elastic and micropolar theories. In order to obtain more realistic values of the ratio $\mu_{c} / \mu^{R}$, a proper inversion process will be performed in future studies. For our illustration purposes we will assume two different values for the ratio $\mu_{c} / \mu^{R}=[0.1,0.5]$. The resulting asymmetric moment tensors are given in Table 1.

To analyze differences in seismograms using the linear elastic and micropolar theories we run numerical simulations of global wave propagation using the freely available package SPECFEM3D GLOBE (Komatitsch \& Tromp, 1999). The numerical package solves the linear elastic equations of motion using the spectral-element method (Komatitsch \& Vilotte, 1998) and we take into account micropolar theory in the rupture area only by implementing an asymmetric moment tensor in the numerical code. Outside the rupture area, only the conventional elastic theory is considered.

Figure 6 shows the predicted T-component seismograms, with a dominant period of $20 \mathrm{~s}$, for the different asymmetric moment tensors presented in Table 1. We can observe differences for the seismograms for symmetric and asymmetric moment tensors in certain directions. Between the two cases of $90^{\circ}$ distance, some wave arrivals show large discrepancies compared with the waveforms predicted by the symmetric moment tensor. These differences are directly related to the computed value of the ratio $\mu_{c} / \mu^{R}$, which serves as a weighting factor of the asymmetric contribution to the total asymmetric seismic moment tensor [see Eq. (42)].

\begin{tabular}{|c|c|c|c|}
\hline Moment tensor component & \multicolumn{3}{|c|}{ Values in $10^{20} \mathrm{~N} \mathrm{~m}$} \\
\hline$M^{\mathrm{sym}}=M^{\mathrm{DC}}+M^{\mathrm{CLVD}}+M^{\mathrm{ISO}}$ & {$\left[\begin{array}{c}3.56 \\
-1.14 \\
4.34\end{array}\right]$} & $\begin{array}{cc}-1.14 & 4.34 \\
1.69 & -2.0 \\
-2.04 & -5.2\end{array}$ & $\left.\begin{array}{l}4 \\
04 \\
25\end{array}\right]$ \\
\hline$M^{\text {skew }}\left(\mu_{c} / \mu^{R}=0.1\right)$ & {$\left[\begin{array}{c}0.0 \\
0.000426 \\
-0.450\end{array}\right.$} & $\begin{array}{c}-0.000426 \\
0.0 \\
0.212\end{array}$ & $\begin{array}{c}0.450 \\
-0.212 \\
0.0\end{array}$ \\
\hline$M^{\text {skew }}\left(\mu_{c} / \mu^{R}=0.5\right)$ & {$\left[\begin{array}{c}0.0 \\
0.00213 \\
-2.25\end{array}\right.$} & $\begin{array}{c}-0.00213 \\
0.0 \\
1.06\end{array}$ & $\left.\begin{array}{c}2.25 \\
-1.06 \\
0.0\end{array}\right]$ \\
\hline
\end{tabular}

Table 1

Micropolar seismic moment tensor of the Kaikoura earthquake 


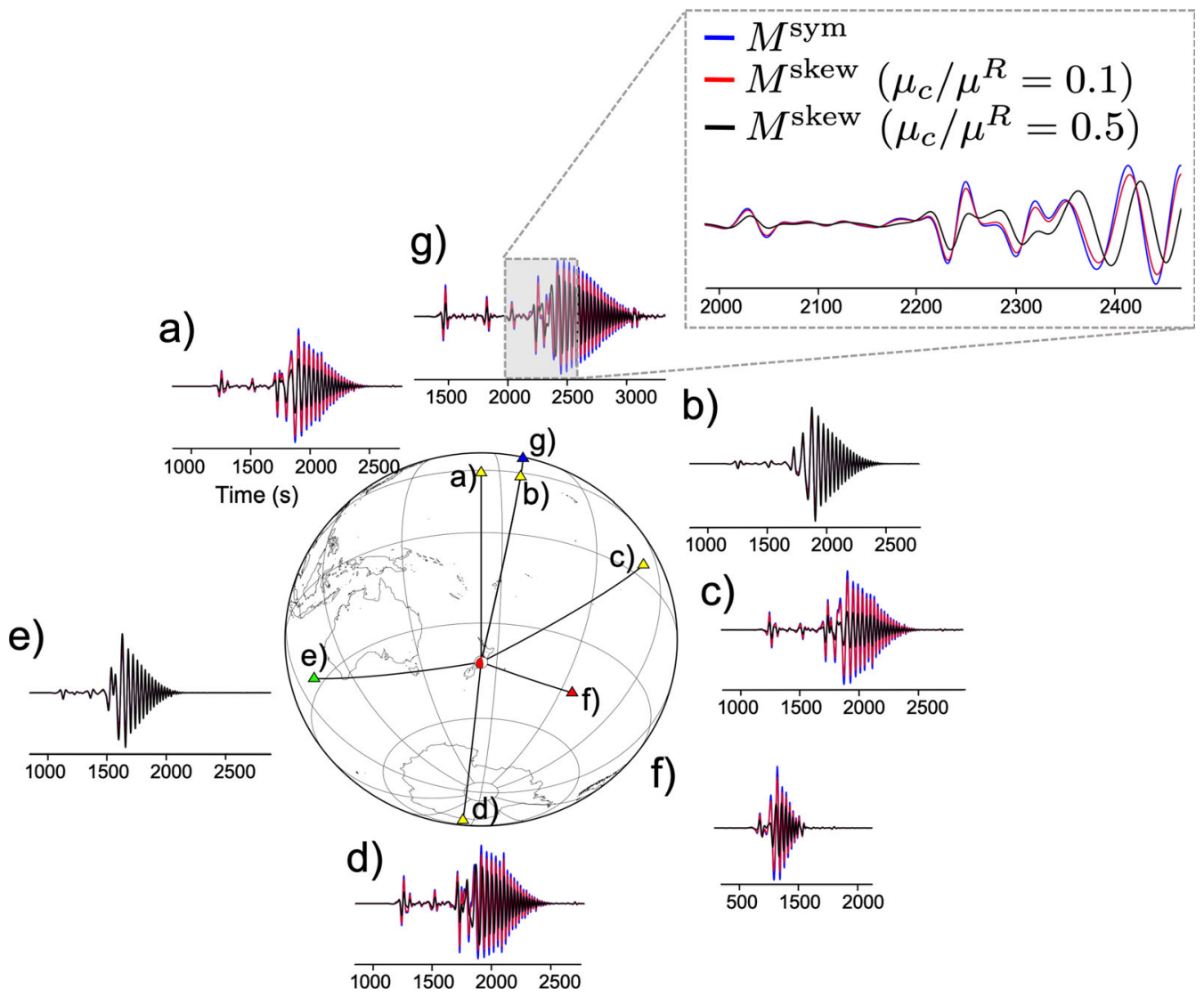

Figure 6

Global numerical T-component seismograms predicted using the spectral-element code SPECFEM3D GLOBE (Komatitsch \& Vilotte, 1998) for the Kaikōura earthquake with a dominant period of $20 \mathrm{~s}$ computed using symmetric $\left(M^{\text {sym }}\right)$ and asymmetric moment tensors $\left(M^{\text {skew }}\right)$. Two different values of the ratio $\mu_{c} / \mu^{R}$ have been tested for the calculation of the micropolar moment tensor. The seismograms are presented at various distances a-d $70^{\circ}$, e $60^{\circ}, \mathbf{f} 30^{\circ}, \mathbf{g ~} 90^{\circ}$ ) and directions

We recall that the purpose of this section is to show that the micropolar moment tensor contribution can have an observable effect on seismic waveforms at teleseismic distances. Since we observe differences in seismic waveforms predicted by symmetric and asymmetric moment tensors, more accurate computations of the micropolar moment tensor can be obtained in the future using full-waveform inversion techniques (Fichtner et al., 2006; Tromp et al., 2005).

The strain deformation observed in the North and South Islands can be computed using the following expressions

$$
\begin{aligned}
\bar{\varepsilon}_{i j}^{\text {Total }} & =\frac{1}{2 \mu \Delta V} \sum_{x=1}^{N} \operatorname{sym}\left(M_{i j}^{\text {Total }}\right)^{(x)}, \\
\overline{\mathbf{W}}_{i j}^{\text {Total }} & =\frac{1}{2 \mu_{c} \Delta V} \sum_{x=1}^{N} \operatorname{skew}\left(M_{i j}^{\text {Total }}\right)^{(x)},
\end{aligned}
$$

where $M_{i j}^{\text {Total }}$ denotes the total moment tensor defined in Eq. (45) and the operator $(\operatorname{sym} X)_{i j}=\frac{1}{2}\left(X_{i j}+X_{j i}\right)$ denotes the symmetric part of $X$ and $(\text { skew } X)_{i j}=$ $\frac{1}{2}\left(X_{i j}-X_{j i}\right)$ denotes the skew-symmetric part of $X$ with $X$ being a second-order tensor. We illustrate Fig. $5 b$ the retrieved rotational field from Eq. (46) considering the Kaikōura event only. This is possible because now the seismic moment tensor is asymmetric, no rotational field is retrieved with conventional symmetric moment tensors. This 
rotational field can be in the future compared with GPS data for instance.

Similar expressions to Eq. (46) have been found by Legrand (2003) with the difference in the rotational deformation $\overline{\mathbf{W}}_{i j}$ divided by $\mu$ instead of the Cosserat couple modulus $\mu_{c}$. Expressions given by Legrand (2003) are found assuming Molnar's asymmetric moment tensor Eq. (19). Comparing the inelastic deformation predicted by Kostrov in Eq. (17) and the one predicted by micropolar theory (46), we can observe that Kostrov's is a case of micropolar theory where the antisymmetric part of the moment tensor has been neglected.

\section{Discussion and Conclusions}

Micropolar theory is today widely applied for the description of localization in faults (Addessi, 2014; Brandes \& Tanner, 2020; Collins-Craft et al., 2020; Rattez, 2017; Rattez et al., 2018a, b; Regenauer-Lieb et al., 2013; Stefanou et al., 2017; Sulem et al., 2011; Veveakis et al., 2012, 2013). In particular the recent book from Žalohar (2018) as well as in the recent works of Rattez et al. (2018a, b) and Collins-Craft et al. (2020) a new approach of the physics of earthquakes based on micropolar theory is developed. In his model, blocks of rocks bounded by fault planes can slide one along another and they can also rotate. Deformation of the faulted rocks in the Earth's crust is described by translational and rotational movements. The size of the rotating blocks defines the Cosserat characteristic length of the crust (Žalohar, 2018). Additionally, fault reactivation is considered to be controlled by a symmetric macro-strain tensor, with large-scale deformation, and a skewed-symmetric relative micro-strain tensor, which describes a local micro-rotations of blocks between faults (Žalohar \& Vrabec, 2010).

At the present time, the physical interpretation of the Cosserat couple modulus $\mu_{c}$ has strongly prevented the application of the dynamic equations of micropolar theory in seismology (Abreu et al., 2017). To overcome this problem we have employed Muhlhaus's theory (Mühlhaus, 1990, 1993; Mühlhaus \& Triantafyllidis, 1987; Mühlhaus \& Vardoulakis, 1986; Papamichos et al., 1990; Sulem \& Vardoulakis,
1995). This theory relates micropolar wave propagation to conventional transverse isotropic media, and it has the advantage that no long-wavelength averaging of the elastic parameters of a layered material must be taken into account like other theories used in the geophysical context Backus (1962). The Cosserat couple modulus $\mu_{c}$ therefore is defined as the difference between Voigt and Reuss moduli [see Eq. (37)]. Using this interpretation, values of the Cosserat couple modulus $\mu_{c}$ are simply a measure of the actual elastic anisotropy observed in the field (Hill, 1952).

Using Muhlhaus's theory allows us to formulate a micropolar seismic moment tensor that reconciles discrepancies between previously introduced asymmetric moment tensors by Molnar (1983), Twiss et al. (1993) and Chapman and Leaney (2019). This allows us to include coseismic clock rotations in the description of the seismic wave propagation in a simple and straightforward way (see Fig. 2). This is done for the first time, and the calculation of the asymmetric micropolar moment tensor only requires the introduction of a single elastic constant: the Cosserat couple modulus $\mu_{c}$, which is again physically understood in terms of seismic anisotropy.

We have proposed a methodology to find the micropolar elastic parameters required to compute the asymmetric moment tensor by combining theories proposed by Postma (1955) and Mühlhaus (1993) and Papamichos et al. (1990). We have computed synthetic global seismograms using the numerical package SPECFEM3D GLOBE (Komatitsch \& Tromp, 1999) for the Kaikōura earthquake. In particular, different studies have shown that this complex event has produced coseismic block rotations (Hamling et al., 2017; Shi et al., 2019; Wang et al., 2020) and we have included, for the first time, these effects on the numerical predicted waveforms by including a micropolar moment tensor. These waveforms have shown differences compared to the those obtained using a conventional symmetric moment tensor. This opens a new avenue for inverting for asymmetric moment tensors using seismic waveforms as routinely done in seismological studies for symmetric moment tensors (e.g. Dahm \& Krüger, 2014). The advantage of including asymmetric moment tensors in the description of seismic events is 
that we include better physics since we can model coseismic block rotations in the moment tensor produced by single events. We recall that this cannot be done with conventional symmetric moment tensors. Following Chapman and Leaney (2019), we reiterate that in an inverse problem it is better to include the extra parameters (force and torque) and determine whether they are necessary from the data rather than eliminate them with a priori assumptions, in particular the asymmetric part of the seismic moment tensor.

The future challenges are several. The adequate visual representation of an asymmetric moment tensor needs to be developed, which requires a further study. The theory of full-waveform inversion for the asymmetric moment tensor, as previously done for the centroid moment tensor (Kim et al., 2011), must also be developed. This will allow us to routinely study crustal block rotations that are observed in many places on Earth, for instance, the San Andreas fault system in southern California (Nicholson et al., 1986a, b; Schreurs, 1994), eastern Iran (Freund, 1970), New Zealand (Roberts, 1995; Ron et al., 1984; Walcott, 1984; Wang et al., 2020), the Dead Sea transform (Pichon \& Gaulie, 1988; Ron \& Eyal, 1985; Ron et al., 1990), the Mojave desert California (Garfunkel, 1974; Schermer et al., 1996), the Sierran microplate, SE California (Lewis et al., 2007) and the Sumatra (McCaffrey et al., 2002; Prawirodirdjo et al., 1997).

\section{Acknowledgements}

R.A. acknowledges initial comments of Jeroen Tromp on micropolar media, Christine Thomas for giving feedback on the manuscript and continuous, amenable and fruitful conversations with Robert Twiss. Maps were drawn with GMT (Wessel \& Smith, 1991), faults of the were obtained from the New Zealand active faults database (Langridge et al., 2016) and the seismic moment tensor was obtained from the CMT catalogue (Ekström et al., 2012). We acknowledge comments from Ioannis Stefanou that helped to improve the quality of the manuscript.

\section{Funding}

Open Access funding enabled and organized by Projekt DEAL.Open Access This article is licensed under a Creative Commons Attribution 4.0 International License, which permits use, sharing, adaptation, distribution and reproduction in any medium or format, as long as you give appropriate credit to the original author(s) and the source, provide a link to the Creative Commons licence, and indicate if changes were made. The images or other third party material in this article are included in the article's Creative Commons licence, unless indicated otherwise in a credit line to the material. If material is not included in the article's Creative Commons licence and your intended use is not permitted by statutory regulation or exceeds the permitted use, you will need to obtain permission directly from the copyright holder. To view a copy of this licence, visit http:// creativecommons.org/licenses/by/4.0/.

Publisher's Note Springer Nature remains neutral with regard to jurisdictional claims in published maps and institutional affiliations.

\section{REFERENCES}

Abreu, R., Durand, S., \& Thomas, C. (2018). The asymmetric seismic moment tensor in micropolar media. Bulletin of the Seismological Society of America, 108(3A), 1160-1170.

Abreu, R., Kamm, J., \& Reiß, A.-S. (2017). Micropolar modelling of rotational waves in seismology. Geophysical Journal International, 210(2), 1021-1046.

Addessi, D. (2014). A 2D Cosserat finite element based on a damage-plastic model for brittle materials. Computers and Structures, 135, 20-31.

Aki, K., \& Richards, P. G. (2002). Quantitative Seismology (2nd ed.). University Science Books.

Allmendinger, R. W., Gephart, J. W., \& Marrett, R. A. (1989). Notes on fault slip analysis. Geological Society of America Short Course, 66.

Alonso-Marroquin, F., Vardoulakis, I., Herrmann, H. J., Weatherley, D., \& Mora, P. (2006). Effect of rolling on dissipation in fault gouges. Physical Review E, 74(3), 031306.

Backus, G. E. (1962). Long-wave elastic anisotropy produced by horizontal layering. Journal of Geophysical Research, 67(11), 4427-4440.

Backus, G., \& Mulcahy, M. (1976). Moment tensors and other phenomenological descriptions of seismic Sources-I. 
Continuous displacements. Geophysical Journal of the Royal Astronomical Society, 46(2), 341-361.

Backus, G., \& Mulcahy, M. (1976). Moment tensors and other phenomenological descriptions of seismic sources-II. Discontinuous displacements. Geophysical Journal of the Royal Astronomical Society, 47(2), 301-329.

Ben-Zion, Y. (2003). Key formulas in earthquake seismology. In W. H. Lee, H. Kanamori, P. Jennings, \& C. Kisslinger (Eds.), International Handbook of Earthquake and Engineering Seismology_Part B (pp. 1857-1875). Elsevier.

Biot, M. A. (1965). Mechanics of Incremental Deformations. Wiley.

Brandes, C., \& Tanner, D. C. (2020). Fault mechanics and earthquakes. In C. Brandes \& D. C. Tanner (Eds.), Understanding Faults: Detecting, Dating and Modeling (pp. 11-80). Elsevier.

Chapman, C. (2010). Fundamentals of Seismic Wave Propagation. Cambridge University Press.

Chapman, C., \& Leaney, S. (2019). Seismic sources at discontinuities: unambiguous moment and potency components. Geophysical Journal International, 216(3), 2004-2016.

Chung, D., \& Buessem, W. (1967). The elastic anisotropy of crystals. Journal of Applied Physics, 38(5), 2010-2012.

Collins-Craft, N. A., Stefanou, I., Sulem, J., \& Einav, I. (2020). A Cosserat Breakage Mechanics model for brittle granular media. Journal of the Mechanics and Physics of Solids, 141, 103975.

Dahlen, F., \& Tromp, J. (1998). Theoretical Global Seismology. Princeton University Press.

Dahm, T., \& Krüger, F. (2014). Moment tensor inversion and moment tensor interpretation. New Manual of Seismological Observatory Practice 2 (NMSOP-2) (pp. 1-37). Deutsches GeoForschungsZentrum GFZ.

Ekström, G., Nettles, M., \& Dziewoński, A. (2012). The global CMT project 2004-2010: Centroid-moment tensors for 13,017 earthquakes. Physics of the Earth and Planetary Interiors, 200, $1-9$.

Eringen, C. (1980). Mechanics of Continua. Wiley.

Fichtner, A., Bunge, H.-P., \& Igel, H. (2006). The adjoint method in seismology: I. Theory. Physics of the Earth and Planetary Interiors, 157(1-2), 86-104.

Freund, R. (1970). Rotation of strike slip faults in Sistan, southeast Iran. The Journal of Geology, 78(2), 188-200.

Garfunkel, Z. (1974). Model for the late Cenozoic tectonic history of the Mojave Desert, California, and for its relation to adjacent regions. Geological Society of America Bulletin, 85(12), 1931-1944.

Garfunkel, Z., \& Ron, H. (1985). Block rotation and deformation by strike-slip faults: 2 . The properties of a type of macroscopic discontinuous deformation. Journal of Geophysical Research: Solid Earth, 90(B10), 8589-8602.

Grekova, E. (2012). Nonlinear isotropic elastic reduced Cosserat continuum as a possible model for geomedium and geomaterials. Spherical prestressed state in the semilinear material. Journal of Seismology, 16(4), 695-707.

Grekova, E. (2012a). Linear reduced Cosserat medium with spherical tensor of inertia, where rotations are not observed in experiment. Mechanics of Solids, 47(5), 538-543.

Grekova, E. (2016). Plane waves in the linear elastic reduced Cosserat medium with a finite axially symmetric coupling between volumetric and rotational strains. Mathematics and Mechanics of Solids, 21(1), 73-93.
Grekova, E., Kulesh, M., \& Herman, G. (2009). Waves in linear elastic media with microrotations, part 2: Isotropic reduced Cosserat model. Bulletin of the Seismological Society of America, 99(2B), 1423-1428.

Hamling, I. J., Hreinsdóttir, S., Clark, K., Elliott, J., Liang, C., Fielding, E., et al. (2017). Complex multifault rupture during the $2016 \mathrm{Mw} 7.8$ Kaikōura earthquake, New Zealand. Science, 356(6334), eaam7194.

Hill, R. (1952). The elastic behavior of a crystalline aggregate. Proceedings of the Physical Society Section A, 65(5), 349.

Jackson, J., \& McKenzie, D. (1988). The relationship between plate motions and seismic moment tensors, and the rates of active deformation in the Mediterranean and Middle East. Geophysical Journal International, 93(1), 45-73.

Kim, Y., Liu, Q., \& Tromp, J. (2011). Adjoint centroid-moment tensor inversions. Geophysical Journal International, 186(1), 264-278.

Kissel, C., \& Laj, C. (2012). Paleomagnetic Rotations and Continental Deformation (Vol. 254). Springer.

Kissel, C., Laj, C., \& Mazaud, A. (1986). First paleomagnetic results from Neocene formations in Evia, Skyros and the Volos region and the deformation of central Aegea. Geophysical Research Letters, 13(13), 1446-1449.

Kissel, C., Laj, C., \& Müller, C. (1985). Tertiary geodynamical evolution of northwestern Greece: Paleomagnetic results. Earth and Planetary Science Letters, 72(2-3), 190-204.

Komatitsch, D., \& Tromp, J. (1999). Introduction to the spectral element method for three-dimensional seismic wave propagation. Geophysical Journal International, 139(3), 806-822.

Komatitsch, D., \& Vilotte, J.-P. (1998). The spectral element method: An efficient tool to simulate the seismic response of 2D and 3D geological structures. Bulletin of the Seismological Society of America, 88(2), 368-392.

Kostrov, V. (1974). Seismic moment and energy of earthquakes, and seismic flow of rock. Izvestiya, Physics of the Solid Earth, 1, 23-44.

Kulesh, M., Grekova, E., \& Shardakov, I. (2009). The problem of surface wave propagation in a reduced Cosserat medium. Acoustical Physics, 55(2), 218-226.

Langridge, R., Ries, W., Litchfield, N., Villamor, P., Van Dissen, R., Barrell, D., et al. (2016). The New Zealand active faults database. New Zealand Journal of Geology and Geophysics, 59(1), 86-96.

Legrand, D. (2003). A short note on the selection of the fault plane, the seismic moment tensor, the strain and rotational tensors, and the gradient of displacement. Bulletin of the Seismological Society of America, 93(2), 946-947.

Lewis, J. C., Twiss, R. J., Pluhar, C. J., Monastero, F. C., Till, A., Roeske, S., et al. (2007). Multiple constraints on divergent strikeslip deformation along the eastern margin of the Sierran microplate, SE California. Special Papers Geological Society of America, 434, 107.

Luyendyk, B. P., Kamerling, M. J., \& Terres, R. (1980). Geometric model for Neogene crustal rotations in southern California. GSA Bulletin, 91(4), 211-217.

Luyendyk, B. P., Kamerling, M. J., Terres, R. R., \& Hornafius, J. S. (1985). Simple shear of southern California during Neogene time suggested by paleomagnetic declinations. Journal of Geophysical Research: Solid Earth, 90(B14), 12454-12466. 
Matsumoto, S., Nishimura, T., \& Ohkura, T. (2016). Inelastic strain rate in the seismogenic layer of Kyushu Island, Japan. Earth, Planets and Space, 68(1), 207.

McCaffrey, R., Stein, S., \& Freymueller, J. (2002). Crustal block rotations and plate coupling. Plate Boundary Zones Geodynamics Series, 30, 101-122.

McKenzie, D., \& Jackson, J. (1986). A block model of distributed deformation by faulting. Journal of the Geological Society, 143(2), 349-353.

Molnar, P. (1983). Average regional strain due to slip on numerous faults of different orientations. Journal of Geophysical Research: Solid Earth, 88(B8), 6430-6432.

Molnar, P., \& Qidong, D. (1984). Faulting associated with large earthquakes and the average rate of deformation in central and eastern Asia. Journal of Geophysical Research: Solid Earth, 89(B7), 6203-6227.

Mühlhaus, H.-B. (1990). Stress and couple stress in a layered half plane with surface loading. International Journal for Numerical and Analytical Methods in Geomechanics, 14(8), 545-563.

Mühlhaus, H.-B. (1993). Continuum models for layered and blocky rock. Analysis and Design Methods (pp. 209-230). Elsevier.

Mühlhaus, H.-B., \& Triantafyllidis, T. (1987). Surface waves in a layered half-space with bending stiffness. In A. Cakmak (Ed.), Ground Motion and Engineering Seismology (Vol. 44, pp. 277-290). Elsevier.

Mühlhaus, H.-B., \& Vardoulakis, I. (1986). Axially-symmetric buckling of the surface of a laminated half space with Bendinh stiffness. Mechanics of Materials, 5(2), 109-120.

Nagahama, H., \& Teisseyre, R. (2000). Micromorphic continuum and fractal properties of faults and earthquakes. In R. Teisseyre \& E. Majewski (Eds.), Earthquake Thermodynamics and Phase Transformations in the Earth's Interior (pp. 425-440). Academic Press.

Nicholson, C., Seeber, L., Williams, P., \& Sykes, L. R. (1986a). Seismic evidence for conjugate slip and block rotation within the San Andreas fault system, southern California. Tectonics, 5(4), 629-648.

Nicholson, C., Seeber, L., Williams, P., \& Sykes, L. R. (1986b). Seismicity and fault kinematics through the eastern transverse ranges, california: Block rotation, strike-slip faulting and lowangle thrusts. Journal of Geophysical Research: Solid Earth, 91(B5), 4891-4908.

Papamichos, E., Vardoulakis, I., \& Mühlhaus, H.-B. (1990). Buckling of layered elastic media: A cosserat-continuum approach and its validation. International Journal for Numerical and Analytical Methods in Geomechanics, 14(7), 473-498.

Pichon, X. L., \& Gaulier, J.-M. (1988). The rotation of Arabia and the Levant fault system. Tectonophysics, 153(1-4), 271-294.

Postma, G. (1955). Wave propagation in a stratified medium. Geophysics, 20(4), 780-806.

Prawirodirdjo, L., Bocl, Y., McCaffrey, R., Genrich, J., Calais, E., Stevens, C., et al. (1997). Geodetic observations of interseismic strain segmentation at the Sumatra subduction zone. Geophysical Research Letters, 24(21), 2601-2604.

Pujol, J. (2003). Elastic Wave Propagation and Generation in Seismology. Cambridge University Press.

Rattez, H. (2017). Couplages Thermo-Hydro-Mécaniques et localisation dans les milieux de Cosserat Application á l'analyse de stabilité $d u$ cisaillement rapide des failles. $\mathrm{PhD}$ thesis, Université Paris-Est.
Rattez, H., Stefanou, I., \& Sulem, J. (2018a). The importance of thermo-hydro-mechanical couplings and microstructure to strain localization in 3D continua with application to seismic faults. Part I: Theory and linear stability analysis. Journal of the Mechanics and Physics of Solids, 115, 54-76.

Rattez, H., Stefanou, I., Sulem, J., Veveakis, M., \& Poulet, T. (2018b). The importance of thermo-hydro-mechanical couplings and microstructure to strain localization in $3 \mathrm{D}$ continua with application to seismic faults. Part II: Numerical implementation and post-bifurcation analysis. Journal of the Mechanics and Physics of Solids, 115, 1-29.

Regenauer-Lieb, K., Veveakis, M., Poulet, T., Wellmann, F., Karrech, A., Liu, J., et al. (2013). Multiscale coupling and multiphysics approaches in earth sciences: Theory. Journal of Coupled Systems and Multiscale Dynamics, 1(1), 49-73.

Roberts, A. P. (1995). Tectonic rotation about the termination of a major strike-slip fault, Marlborough Fault System, New Zealand. Geophysical Research Letters, 22(3), 187-190.

Ron, H., \& Eyal, Y. (1985). Intraplate deformation by block rotation and mesostructures along the Dead Sea transform, northern Israel. Tectonics, 4(1), 85-105.

Ron, H., Freund, R., Garfunkel, Z., \& Nur, A. (1984). Block rotation by strike-slip faulting: Structural and paleomagnetic evidence. Journal of Geophysical Research: Solid Earth, 89(B7), 6256-6270.

Ron, H., Nur, A., \& Eyal, Y. (1990). Multiple strike-slip fault sets: A case study from the Dead Sea Transform. Tectonics, 9(6), 1421-1431.

Schermer, E. R., Luyendyk, B., \& Cisowski, S. (1996). Late Cenozoic structure and tectonics of the northern Mojave Desert. Tectonics, 15(5), 905-932.

Schreurs, G. (1994). Experiments on strike-slip faulting and block rotation. Geology, 22(6), 567-570.

Shi, X., Tapponnier, P., Wang, T., Wei, S., Wang, Y., Wang, X., \& Jiao, L. (2019). Triple junction kinematics accounts for the 2016 Mw 7.8 Kaikoura earthquake rupture complexity. Proceedings of the National Academy of Sciences, 116(52), 26367-26375.

Sibson, R. H. (1984). Roughness at the base of the seismogenic zone: Contributing factors. Journal of Geophysical Research: Solid Earth, 89(B7), 5791-5799.

Slawinski, M. (2010). Waves and Rays in Elastic Continua. World Scientific Publishing Company.

Stefanou, I., Sulem, J., \& Rattez, H. (2017). Cosserat approach to localization in geomaterials. In G. Z. Voyiadjis (Ed.), Handbook of Nonlocal Continuum Mechanics for Materials and Structures (pp. 1-25). Springer.

Sulem, J., Stefanou, I., \& Veveakis, E. (2011). Stability analysis of undrained adiabatic shearing of a rock layer with Cosserat microstructure. Granular Matter, 13(3), 261-268.

Sulem, J., \& Vardoulakis, I. (1995). Bifurcation Analysis in Geomechanics. CRC Press.

Teisseyre, R. (1973). Earthquake processes in a micromorphic continuum. Pure and Applied Geophysics, 102(1), 15-28.

Teisseyre, R. (2008). Friction and fracture induced anisotropy: Asymmetric stresses. In R. Teisseyre, H. Nagahama, \& E. Majewski (Eds.), Physics of Asymmetric Continuum: Extreme and Fracture Processes (pp. 163-169). Springer.

Teisseyre, R. (2011). Why rotation seismology: Confrontation between classic and asymmetric theories. Bulletin of The Seismological Society of America, 101, 1683-1691. 
Teisseyre, R., Gorski, M., \& Teisseyre, K. (2008). Fracture processes: Spin and twist-shear coincidence. In R. Teisseyre, H. Nagahama, \& E. Majewski (Eds.), Physics of Asymmetric Continuum: Extreme and Fracture Processes (pp. 111-122). Springer.

Teisseyre, R., Takeo, M., \& Majewski, E. (2006). Earthquake source asymmetry. Structural Media and Rotation Effects. Springer.

Tromp, J., Tape, C., \& Liu, Q. (2005). Seismic tomography, adjoint methods, time reversal and banana-doughnut kernels. Geophysical Journal International, 160(1), 195-216.

Twiss, R. J. (2009). An asymmetric micropolar moment tensor derived from a discrete-block model for a rotating granular substructure. Bulletin of the Seismological Society of America, 99(2B), 1103-1131.

Twiss, R. J., Protzman, G. M., \& Hurst, S. D. (1991). Theory of slickenline patterns based on the velocity gradient tensor and microrotation. Tectonophysics, 186(3), 215-239.

Twiss, R. J., Souter, B. J., \& Unruh, J. R. (1993). The effect of block rotations on the global seismic moment tensor and the patterns of seismic $\mathrm{P}$ and $\mathrm{T}$ axes. Journal of Geophysical Research: Solid Earth, 98(B1), 645-674.

Twiss, R. J., \& Unruh, J. R. (2007). Structure, deformation, and strength of the Loma Prieta fault, northern California, USA, as inferred from the 1989-1990 Loma Prieta aftershock sequence. Geological Society of America Bulletin, 119(9-10), 1079-1106.

Udias, A., \& Buforn, E. (2018). Principles of Seismology. Cambridge University Press.

Unruh, J. R., Twiss, R. J., \& Hauksson, E. (1996). Seismogenic deformation field in the Mojave block and implications for tectonics of the eastern California shear zone. Journal of Geophysical Research: Solid Earth, 101(B4), 8335-8361.

Vavryčuk, V. (2005). Focal mechanisms in anisotropic media. Geophysical Journal International, 161(2), 334-346.
Veveakis, M., Stefanou, I., \& Sulem, J. (2013). Failure in shear bands for granular materials: Thermo-hydro-chemo-mechanical effects. Géotechnique Letters, 3(2), 31-36.

Veveakis, E., Sulem, J., \& Stefanou, I. (2012). Modeling of fault gouges with Cosserat Continuum Mechanics: Influence of thermal pressurization and chemical decomposition as coseismic weakening mechanisms. Journal of Structural Geology, 38, 254-264.

Walcott, R. (1984). The kinematics of the plate boundary zone through New Zealand: A comparison of short-and long-term deformations. Geophysical Journal International, 79(2), 613-633.

Wang, T., Jiao, L., Tapponnier, P., Shi, X., \& Wei, S. (2020). Space imaging geodesy reveals near circular, coseismic block rotation during the $2016 \mathrm{Mw} 7.8$ Kaikōura earthquake, New Zealand. Geophysical Research Letters, 47(22), e2020GL090206.

Watt, J. P., Davies, G. F., \& O'Connell, R. J. (1976). The elastic properties of composite materials. Reviews of Geophysics, 14(4), 541-563.

Wells, R. E., \& Heller, P. L. (1988). The relative contribution of accretion, shear, and extension to Cenozoic tectonic rotation in the Pacific Northwest. GSA Bulletin, 100(3), 325-338.

Wernicke, B., \& Burchfiel, B. (1982). Modes of extensional tectonics. Journal of Structural Geology, 4(2), 105-115.

Wessel, P., \& Smith, W. H. (1991). Free software helps map and display data. Eos, Transactions American Geophysical Union, 72(41), 441-446.

Woodhouse, J. (1981). The excitation of long period seismic waves by a source spanning a structural discontinuity. Geophysical Research Letters, 8(11), 1129-1131.

Žalohar, J. (2018). The Omega-Theory: A New Physics of Earthquakes. Elsevier.

Žalohar, J., \& Vrabec, M. (2010). Kinematics and dynamics of fault reactivation: The Cosserat approach. Journal of Structural Geology, 32(1), 15-27. 